\title{
Can Ferrroelasticity be Evaluated by Nanoindentation?
}

Wakako Araki ${ }^{\mathrm{a},}$, Xin Wang ${ }^{\mathrm{b}}$, and Alan Atkinson ${ }^{\mathrm{b}}$

${ }^{a}$ Department of Mechanical Engineering, Saitama University, 255 Shimo-Okubo, Sakura-ku, Saitama 3388570, Japan

${ }^{\mathrm{b}}$ Department of Materials, Imperial College London, London SW7 2AZ, UK

*Corresponding author. 255 Shimo-Okubo, Sakura-ku, Saitama 3388570 Japan. Tel.: +81 0488583435.

E-mail address: araki@mech.saitama-u.ac.jp (W Araki)

\begin{abstract}
The present study investigated the possibility of evaluating ferroelastic mechanical characteristics by spherical indentation. Finite element simulation of spherical indentation with a relatively large sphere of a ferroelastic-plastic material was performed using characteristic bulk data of a typical ferroelastic oxide (LaSr-Co-Fe-O). The simulation results showed that the ferroelastic mechanical behaviour cannot be observed in the indentation load vs depth curve, but is clearly observable in the indentation stress vs indentation strain curve, which can be obtained reliably in experiments by estimating the contact radius using load-partial unloading sequences. The method can be reliable when the indentation stress is under the upper ferroelastic critical stress. Therefore, in principle ferroelastic mechanical characteristics could be evaluated by spherical indentation by obtaining the indentation stress vs indentation strain curve using partial unloading to estimate the contact radius, although the requirements are very difficult to satisfy in actual experiments.
\end{abstract}

Keywords

Ferroelasticity; Nanoindentation; Finite element method; Mechanical properties; LSCF 


\section{Graphical Abstract}

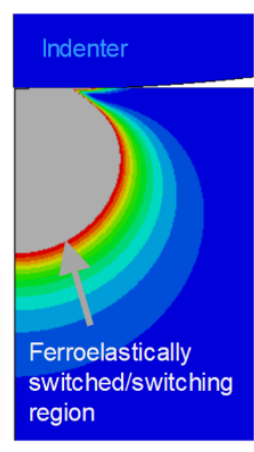

Spherical indentation

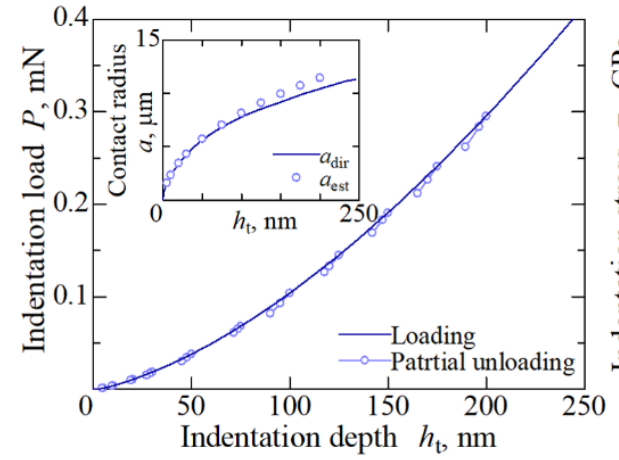

No indication of ferroelasticity in $P$ vs $h_{\mathrm{t}}$ curve

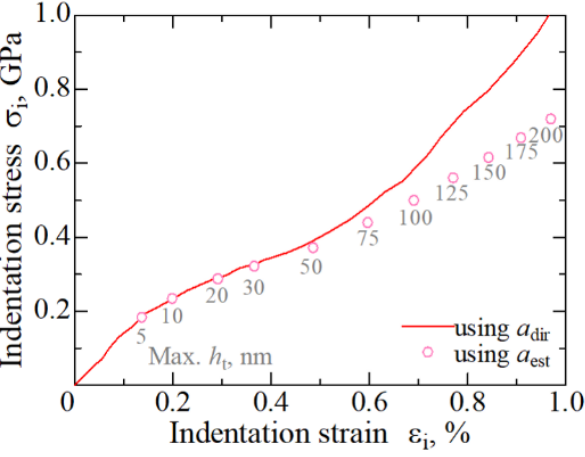

Clear indication of ferroelasticity in $\sigma_{\mathrm{i}}$ VS $\varepsilon_{\mathrm{i}}$ curve 


\section{Introduction}

Unlike conventional ceramics, ferroelastic ceramics exhibit a strongly nonlinear behaviour in their mechanical response, which can be observed in stress-strain curves obtained in a uniaxial compressive test. Many oxides exhibit this behaviour and are used for their other properties in technology applications. For example, some of the ceramic materials that have been optimised in terms of performance for application in Solid Oxide Fuel Cells (SOFC), such as scandia-stabilized zirconia electrolytes and lanthanum cobaltitebased cathodes, have been reported to have ferroelasticity. ${ }^{1-5}$ The non-linearity in stress-strain response is attributed to a domain switching phenomenon in response to applied mechanical stress. ${ }^{6}$ Crystals showing this behaviour typically show small distortions from a high symmetry parent structure in which the distortions have several equivalent orientations and easy switching between the distortion orientations (e.g. by the small displacement of a single atom from its high symmetry position as for the octahedrally co-ordinated B-site atom in the perovskite structure). In a given domain all the distortions are aligned in a common direction. Switching of the orientation by motion of a domain wall results in a crystal strain in such a way as to amplify the linear elastic strain. The nonlinear mechanical behaviour can be characterised by an initial modulus before domain switching, a critical stress for domain switching, and loading modulus after all domains have switched. ${ }^{1,7}$ In practice it is found that there is not a single stress level at which all domains switch (which would cause a sudden increase in strain), but a range of stresses reflecting a range of switching stresses for different domain walls. The nonlinear mechanical behaviour as well as the domain switching process of ferroelastic materials has been also captured by means of numerical simulations. ${ }^{8,9}$

In previous studies, the mechanical behaviour of ferroelastic $\mathrm{La}_{0.58} \mathrm{Sr}_{0.4} \mathrm{Co}_{0.2} \mathrm{Fe}_{0.8} \mathrm{O}_{3-\delta}$ and $\mathrm{La}_{0.6} \mathrm{Sr}_{0.4} \mathrm{Co}_{0.2} \mathrm{Fe}_{0.8} \mathrm{O}_{3-\delta}$ (LSCF), a material used as an $\mathrm{SOFC}$ cathode, was characterised under uniaxial compression at different temperatures. LSCF exhibited ferroelastic behaviour from room temperature to 1073 $\mathrm{K}$, embracing ferro- to para-elastic and rhombohedral to cubic transition temperatures. A superelastic-like mechanical behaviour with a large hysteresis was also confirmed below room temperature. ${ }^{2}$ (In fact, ferroelastic behaviour is often observed in twinned martensite phase of shape memory alloys, and ferroelasticity of shape memory alloy is known to be associated with martensitic reorientation, whereas 
superelasticity is associated with martensitic transformation. Their mechanical responses similarly exhibit hysteresis loops. ${ }^{10-12}$ )

Mechanical behaviour of materials can be studied not only by macroscopic tests, but also by microscopic techniques such as nanoindentation. The mechanical properties of LSCF have been investigated by nanoindentation in previous studies. ${ }^{13-15}$ Young's modulus and fracture toughness were determined using standard analysis assuming a linear elastic response, and no anomaly that could be attributed to ferroelastic behaviour was seen in the indentation load vs depth curves. One possible reason why the ferroelastic characteristics were not observed in the previous indentation studies could be that the stress levels caused by the indentation were too high for the detection of domain switching. Similarly, no superelastic characteristics, i.e., stress-strain relationship, have been successfully observed by indentation techniques. ${ }^{16-20}$ This suggests that the intrinsic characteristics of a ferroelastic material cannot be captured by indentation. On the other hand, analytical models of elasto-plastic indentation have been well developed to determine mechanical characteristics, i.e., analysing indentation stress-strain curves from spherical indentation to determine Young's modulus and yield stress. Thus, ferroelastic characteristics might be observable by applying the same methods developed for analysing spherical indentation of elasto-plastic materials to ferroelastic ones.

The present study therefore examines the possibility of evaluating ferroelastic characteristics by means of spherical indentation. First, the examination is performed by finite element simulation of the indentation process. Since one of the reasons why ferroelastic characteristics have not been observed in the previous studies could be the high stresses caused by indentation; thus, the present study employs spherical indentation with a relatively large sphere. Spherical indentation of a ferroelastic-perfectly plastic material was simulated by the finite element method, in which a material model for superelastic material with a shape-memory effect was employed. Then, the simulated load vs indentation curves were analysed using the method suggested by Field and Swain for determination of elasto-plastic characteristics. For comparison, both elastic and elastoplastic indentations were simulated. The present discussion would also be applicable to superelastic materials. Based on the simulated results, a spherical indentation experiment was performed on dense samples of LSCF. 


\section{Theoretical}

A simple approach for analysing elasto-plastic spherical indentation was proposed by Field and Swain, ${ }^{21,22}$ and employs loading-unloading sequences. In the present study, this approach was applied to analyse the simulated spherical indentation of a ferroelastic-plastic material to investigate whether the ferroelastic behaviour could be detected in the resulting indentation stress-strain curves. The analysis method for a loading-unloading sequence is illustrated in Fig. 1 and briefly explained below. More details can be found in the literature. ${ }^{21,22}$

For purely elastic indentation with a spherical indenter of radius $R$, a relationship between load $P$ and elastic depth $\delta$ can be given by ${ }^{23}$

$$
\delta=\left(\frac{9}{16 R E^{* 2}}\right)^{1 / 3} P^{2 / 3}=q P^{2 / 3}
$$

In this expression $q$ is a system constant (depending on the two materials in contact) and $E^{*}$ is an effective elastic modulus given by

$$
\frac{1}{E^{*}}=\frac{1-v^{2}}{E}+\frac{1-v_{\mathrm{i}}^{2}}{E_{\mathrm{i}}}
$$

where $v, E, v_{\mathrm{i}}$, and $E_{\mathrm{i}}$ are Poisson's ratio and Young's modulus of sample and indenter, respectively. Accordingly, Young's modulus of an elastic sample $E$ can be determined by Eqs. (1) and (2), assuming the value of $v$.

For elasto-plastic indentation, the total depth $h_{\mathrm{t}}$ can be considered as a sum of the elastic component $h_{\mathrm{e}}$ and plastic component $h_{\mathrm{p}}$ caused during the loading as illustrated in Fig. 1(b), i.e.,

$$
h_{\mathrm{t}}=h_{\mathrm{e}}+h_{\mathrm{p}}=\frac{\delta}{2}+h_{\mathrm{p}}
$$

The total depth $h_{\mathrm{t}}$ under the load $P$ can be also expressed by a sum of the elastically recovered component $\delta^{*}$ and residual depth $h_{\mathrm{r}}$ after full unloading, i.e.

$$
h_{\mathrm{t}}=\delta^{*}+h_{\mathrm{r}}=q P_{\mathrm{t}}^{2 / 3}+h_{\mathrm{r}}
$$

In the partially unloaded state shown in Fig. 1 , the total depth $h_{\mathrm{s}}$ under the load $P_{\mathrm{s}}$ is given by

$$
h_{\mathrm{s}}=\delta_{\mathrm{s}}^{*}+h_{\mathrm{r}}=q P_{\mathrm{s}}^{2 / 3}+h_{\mathrm{r}}
$$

where $\delta_{\mathrm{s}}{ }^{*}$ is the elastically recovered component from the partially unloaded state. The residual depth $h_{\mathrm{r}}$ 
therefore can be estimated by the partial unloading process using Eqs. (4) and (5) as

$$
h_{\mathrm{r}}=\frac{h_{\mathrm{s}}\left(P_{\mathrm{t}} / P_{\mathrm{s}}\right)^{2 / 3}-h_{\mathrm{t}}}{\left(P_{\mathrm{t}} / P_{\mathrm{s}}\right)^{2 / 3}-1}
$$

As $\delta^{*}$ can be considered to be equal to $\delta$, and the plastic component $h_{\mathrm{p}}$ can be expressed by

$$
h_{\mathrm{p}}=\frac{h_{\mathrm{t}}+\mathrm{h}_{\mathrm{r}}}{2}
$$

The contact radius between the indenter and sample $a$ illustrated in Fig. 1(b) can then be estimated from $h_{\mathrm{p}}$ as

$$
a=\left(2 R h_{\mathrm{p}}-h_{\mathrm{p}}^{2}\right)^{1 / 2}
$$

The composite modulus $E^{*}$ can then be determined by using $a$ as

$$
E^{*}=\frac{3 P}{4 a \delta} \quad \text { (9), }
$$

and subsequently Young's modulus of the sample $E$ can be determined. Indentation stress $\sigma_{\mathrm{i}}$ and indentation strain $\varepsilon_{\mathrm{i}}$ can be also derived using $a$ as follows

$$
\sigma_{\mathrm{i}}=\frac{P}{\pi a^{2}} \quad(10),
$$

and

$$
\varepsilon_{\mathrm{i}}=\frac{4 a}{3 \pi R} \quad \text { (11). }
$$

\section{Simulation}

\subsection{Finite element analysis}

A commercial software (ABAQUS 6.14) was used for the present finite element analysis. Figure 2 shows the axisymmetric simulation model used in this study, which consists of two parts: a disk-shaped flat planar sample and a hemi-spherical indenter. The disk sample has a radius and height of $25 \mathrm{~mm}$, whilst the spherical indenter has a radius of $500 \mu \mathrm{m}$ in the base case. The boundary condition at the bottom surface of the disk is illustrated in Fig. 2(a). The model was meshed into 108407 elements (326430 nodes) using eightnode axisymmetric elements (CAX8). It should be noted that the sample region near the contact with the indenter was meshed very finely as shown in Fig. 2(b), not only for analysis convergence, but also for direct 
evaluation of contact radius as explained below. (The preliminary analysis using elastic material property confirmed the validity of the present model in terms of geometry and mesh by comparing the indentation depth, indentation load, and the contact area obtained by the simulation and Hertzian contact theory.)

The sample material was assumed to be either elastic, elasto-plastic, or ferroelastic-plastic, whilst the indenter was elastic diamond. The material properties used in the simulation are summarised in Table 1 and Fig. 3, where the ferroelastic-plastic properties were taken from the experimental data of LSCF obtained in the previous studies, ${ }^{1,2}$ namely, initial modulus $E_{\mathrm{A}}$, lower and upper critical stresses $\sigma_{\mathrm{L}}$ and $\sigma_{\mathrm{U}}$, loading modulus $E_{\mathrm{B}}$, and yield stress $\sigma_{\mathrm{Y}} .^{7}$ The loading-unloading sequence was simulated for elasto-plastic and ferroelastic-plastic materials, where loading subsequently followed by partial unloading as Fig. 1(a) was simulated. For ferroelastic-plastic simulation, two different material models for superelastic (or hyperelastic in ABAQUS) material were used, i.e., Nitinol and Marlow models. (Ferroelasticity was assumed to be a part of category of superelasticity with delayed recovery in this study, considering the similarities in their mechanical bahaviours.) The Nitinol model can reproduce the shape memory effect (shown as dashed lines in Fig. 3) and so was used for the loading-unloading simulations, whilst the Marlow model was used only for the monotonic loading simulations but up to higher load for better analysis stability. The ferroelastic backswitching deformation was assumed not to occur in the partial unloading simulation with the Nitinol model.

In the loading simulation, a displacement up to a maximum of $1 \mu \mathrm{m}$ with stepwise increments was applied to the top surface of the half indenter. The relationship between the indentation load $P$ and the total penetration depth $h_{\mathrm{t}}$ was obtained, where $P$ was calculated as the total repulsive load on the top surface of the indenter, and $h_{\mathrm{t}}$ was equal to the applied displacement. The contact radius between the sample and indenter $a_{\mathrm{dir}}$ was also obtained in the simulation, where the contact edge was defined as where the contact stress on the sample surface became non-zero. In the partial unloading simulation, $20 \%$ of the maximum displacement was unloaded and the contact radius $a_{\text {est }}$ was estimated according to Eqs. (6)-(8). The indentation stress $\sigma_{\mathrm{i}}$ and strain $\varepsilon_{\mathrm{i}}$ curves were then obtained using both $a_{\mathrm{dir}}$ and $a_{\mathrm{est}}$ in Eqs. (10) and (11), and the results were compared. Although stress vs strain relationships of ferroelastic materials are known to depend on strain rate, ${ }^{2,24}$ no strain-rate dependence was considered in the simulation. 


\subsection{Simulation results}

Figure 4(a) shows the simulated relationships between load $P$ and the total depth $h_{\mathrm{t}}$ of the elastic, elastoplastic, and ferroelastic-plastic materials, where the ferroelastic-plastic simulation was performed by both Nitinol (N) and Marlow (M) models. (Note that the curves of the elastic and elasto-plastic materials are overlapped, whilst the ones of the ferroelastic-plastic materials are overlapped each other.) In all cases the load shows a monotonic smoothly increasing trend with $h_{\mathrm{t}}$, although the input material properties are quite different. The inset of Fig. 4(a) shows the relationship between the total depth $h_{\mathrm{t}}$ and the contact radius $a_{\mathrm{dir}}$, which is directly evaluated from the simulation result using the contact stress criterion. All the radii $a_{\mathrm{dir}}$ show a similar increasing trend with $h_{\mathrm{t}}$ regardless of the material model used. Figure 4 (b) shows the indentation stress $\sigma_{\mathrm{i}}-$ strain $\varepsilon_{\mathrm{i}}$ curves derived using $a_{\mathrm{dir}}$. The elastic material has a linear relationship as expected, and the estimated $E^{*}$ of the sample from the simulation was $145 \mathrm{GPa}$ (i.e., the estimated $E$ of the sample was 151 GPa with the deviation from the input value of $0.7 \%$ ). The elasto-plastic material simulation also shows a linear behaviour with $E^{*}=144 \mathrm{GPa}$ under lower stress (i.e., the estimated $E=150 \mathrm{GPa}$ ) and shows onset of plasticity at the stress above $\sigma_{\mathrm{i}}=\sim 5.5 \mathrm{GPa}$. Plasticity is known to be observed in spherical indentation at indentation stress in the range of approximately $1.1 \sigma_{\mathrm{Y}}$ (start of plastic flow) and $3.0 \sigma_{\mathrm{Y}}$ (complete flow). ${ }^{25} \mathrm{In}$ the present case, the indentation yield stress $\sigma_{\mathrm{i}}=5.5 \mathrm{GPa}$ is equivalent to $1.2 \sigma_{\mathrm{Y}}$, which is a reasonable value. (Additional simulation with much larger displacements revealed the indentation stress is saturated at $2.8 \sigma_{\mathrm{Y}}$ at an indentation strain above $10 \%$, which is close to $3.0 \sigma_{\mathrm{Y}}$. Note that this also indicates an estimated saturation hardness of $12.9 \mathrm{GPa}$ in this case.) For the ferroelastic-plastic material simulation, the $\sigma_{\mathrm{i}}-\varepsilon_{\mathrm{i}}$ curve exhibits an initial linear behaviour also with $E^{*}=145 \mathrm{GPa}$ (namely $E=151 \mathrm{GPa}$ ) followed by a strong nonlinearity, which resembles the input material behaviour, and both Nitinol and Marlow models produce a similar behaviour. The indentation critical stress for domain switching from the simulation in Fig. 4(b) appears to be between $200 \mathrm{MPa}$ (lower) and $400 \mathrm{MPa}$ (upper), which are almost double the input critical stresses, i.e., 105 and $237 \mathrm{MPa}$, whilst the corresponding strains are only slightly different. Figure 5 shows the distribution of elastic strain energy density in the deformed region. The grey area is the estimated 
ferroelastically domain-switching or switched region. The critical energy density required for the start of domain switching process used for this criterion was $42 \mathrm{~kJ} / \mathrm{m}^{3}$, which was calculated based on uniaxial compression data up to the lower critical stress. The indentation yield stress from the simulation, $\sigma_{\mathrm{i}}$, is approximately $5.5 \mathrm{GPa}$, i.e., $1.2 \sigma_{\mathrm{Y}}$. The results suggest that the ferroelastic behaviour could be observed in principle by using not $P$ vs $h_{\mathrm{t}}$, but through the $\sigma_{\mathrm{i}}-\varepsilon_{\mathrm{i}}$ relationship.

Figure 6 shows the $P-h_{\mathrm{t}}$ curve of the elasto-plastic material with the loading-unloading sequence, where the unloadings of $10 \sim 20 \%$ from five different maximum displacements were simulated. The inset of Fig. 6(a) compares the relationship between the total depth $h_{\mathrm{t}}$ and the contact radius $a_{\mathrm{dir}}$ of the elasto-plastic material, where $a_{\mathrm{dir}}$ is directly obtained from the simulation result and $a_{\mathrm{est}}$ is indirectly estimated by using the partial unloading data. The contact radius $a_{\text {est }}$ appears to be consistent to $a_{\text {dir. }}$ Figure 6(b) compares the $\sigma_{\mathrm{i}}-$ $\varepsilon_{\mathrm{i}}$ curves of the elasto-plastic material using $a_{\mathrm{dir}}$ and $a_{\text {est. }}$ The $\sigma_{\mathrm{i}}-\varepsilon_{\mathrm{i}}$ curve estimated by $a_{\text {est }}$ agrees well with the direct simulation result. (The maximum deviation from the theoretical value is $0.7 \%$ in terms of $E^{*}$ using Eq. (9).) These results confirm the validities of the estimation technique with the loading-unloading sequence and the present simulation.

Figure 7(a) shows the simulated $P$ vs $h_{\mathrm{t}}$ curves of the ferroelastic-plastic material with the loadingunloading sequences, where only the Nitinol model was used up to $\varepsilon_{\mathrm{i}}=\sim 1 \%$. The inset of Fig. 7(a) compares the contact radii $a_{\mathrm{dir}}$ and $a_{\text {est }}$, where $a_{\mathrm{dir}}$ is directly obtained from the simulation and $a_{\mathrm{est}}$ is indirectly estimated by using the simulated unloading data. The radii $a_{\mathrm{dir}}$ and $a_{\mathrm{est}}$ again agree well with each other for smaller $h_{\mathrm{t}}$, but differ significantly for larger $h_{\mathrm{t}}$. Figure 7 (b) compares the $\sigma_{\mathrm{i}}$ vs $\varepsilon_{\mathrm{i}}$ curves of the ferroelastic material obtained by $a_{\mathrm{dir}}$ and $a_{\mathrm{est}}$. The curves derived from $a_{\mathrm{dir}}$ and $a_{\mathrm{est}}$ are reasonably consistent for lower $\sigma_{\mathrm{i}}$, whereas they become inconsistent above the upper indentation critical stress ( $400 \mathrm{MPa})$, which is simply attributed to the difference between $a_{\mathrm{dir}}$ and $a_{\mathrm{est}}$. This result indicates the possibility of obtaining $\sigma_{\mathrm{i}}$ vs $\varepsilon_{\mathrm{i}}$ curve of a ferroelastic material by using $a_{\text {est }}$ based on the loading-unloading sequences but only below the upper indentation critical stress.

\subsection{Discussion}


The simulation results of spherical indentation show that ferroelasto-plastic mechanical behaviour (in a representative ferroelastic perovskite) cannot be observed in an indentation load vs depth curve. However, it can be seen in a plot of indentation stress vs indentation strain. The simulation also demonstrated that a suitable method for measuring the indentation stress vs strain curve is the loading and partial-unloading sequence suggested by Field and Swain to estimate the contact area, although it becomes less reliable when the indentation stress is above the upper critical stress (at which almost all domains are aligned).

This is because of the different elastic modulus after domain alignment. The estimation of the contact radius $a_{\text {est }}$ by the partial unloading sequence is based on the assumption that the elastic deformation during the loading $\delta$ and the elastic recovery during the unloading $\delta^{*}$ are controlled by the same elastic modulus. This is a reasonable assumption for elasto-plastic materials, and its validity was confirmed in the simulations as shown in Fig. 6. However, for ferroelastic-plastic (or simply ferroelastic) materials, the actual $\delta^{*}$ during the unloading will be smaller than $\delta$, because the Young's modulus of the ferroelastic material during the unloading is higher than the one during the loading due to the aligned ferroelastic domains. (A simulation of uniaxial compression confirmed this "stiffening" effect when the applied stress is higher than lower critical stress, as shown in Fig. 3.) This inaccurate assumption leads to $a_{\text {est }}$ being higher than $a_{\text {dir }}$ as shown in the inset of Fig. 7(a), and accordingly leads to a lower estimate of $\sigma_{\mathrm{i}}$ and a higher estimate of $\varepsilon_{\mathrm{i}}$ for a given displacement. (From Eqs. (10) and (11), $\varepsilon_{\mathrm{i}}$ and $\sigma_{\mathrm{i}}$ are proportional to $a$ and $a^{-2}$ respectively.) Nevertheless, the error in $a_{\text {est }}$ is significant only when the indentation stress exceeds the upper critical stress, and so it is still possible to evaluate the initial modulus and critical stresses of ferroelastic behaviour using the loadingunloading sequence.

It should be noted that the present simulation would also be relevant to superelastic materials with shapememory effect. Although a number of studies have investigated these materials by indentation, ${ }^{16-20}$ none has succeeded in revealing its nonlinear mechanical characteristics clearly, because most of them only examined the indentation load vs depth curve. As superelastic materials exhibit generally similar mechanical behaviour to ferroelastic materials but have much larger deformation during the transformation ( $\sim 10$ times), it would be experimentally possible to estimate contact radius and thus the stress-strain relationship (but only up to 
the upper transformation stress) by using the loading-unloading sequence.

The practicality of using this method in actual experiments is now considered. Figure 7(b) shows the maximum indentation depths used for the loading-unloading simulations. It demonstrates that the maximum depth required for the evaluation of ferroelastic properties in LSCF (such as initial modulus, lower critical stress, and upper critical stress) is smaller than $80 \mathrm{~nm}$ (i.e., smaller than $0.4 \mathrm{GPa}$ of indentation stress) for a $0.5 \mathrm{~mm}$ radius indenter. This would be very challenging to achieve in experiments due to effects of surface roughness and unstable initial contact, and is experimentally examined in the following chapter. It should be also worth noting that our additional simulations with smaller spherical indenters ( $25 \mu \mathrm{m}$ and $50 \mu \mathrm{m}$ radius) confirmed that the indentation stress-strain curve should be consistent regardless of indenter size; however, the evaluation of contact radius requires even higher accuracy in depth measurement for indenters of smaller radius.

\section{Experimental}

\subsection{Spherical indentation}

The spherical indentation of a dense polycrystalline LSCF sample was performed using a NanoTest Vantage instrument (Micro Materials, UK). The starting powder, lanthanum strontium cobalt ferrite ( $\mathrm{La}_{0.6} \mathrm{Sr}_{0.4} \mathrm{Co}_{0.2} \mathrm{Fe}_{0.8} \mathrm{O}_{3-\delta}$, AGC Seimi Chemical, Japan) was uniaxially pressed into a disc, which was sintered at $1473 \mathrm{~K}$ for $6 \mathrm{~h}$. The sintered sample was then cut, annealed, and mirror-polished. More details on the sample preparation can be found in the literature. ${ }^{2,26}$ In the indentation test, a relatively large sapphire indenter with radius $500 \mu \mathrm{m}$ (made of a sapphire ball lens) was used, whilst a diamond indenter with radius $25 \mu \mathrm{m}$ was also used for comparison. (It should be noted that the effective tip radius of the diamond indenter was actually determined to be $15 \mu \mathrm{m}$ based on a preliminary test with a reference sample of quartz.) Loadingunloading sequences with increasing maximum loads (up to $500 \mathrm{mN}$ for the $500 \mu \mathrm{m}$ indenter, and $300 \mathrm{mN}$ for $25 \mu \mathrm{m}$, respectively), was applied. In each cycle $50 \%$ of maximum load was unloaded after every loading. Both loading and unloading were applied in $1 \mathrm{~s}$ with a holding period of $1 \mathrm{~s}$ at each maximum load, whilst the load vs total depth curve was recorded in $50 \mathrm{~ms}$. The indentation stress vs indentation strain relationship 
was obtained using the linear part of the unloading curves.

\subsection{Results}

Figure 8(a) shows the load $P$ vs the total depth $h_{\mathrm{t}}$ curve of LSCF obtained using the sapphire indenter with radius $500 \mu \mathrm{m}$. The inset shows the result obtained with the diamond indenter with the nominal radius of 25 $\mu \mathrm{m}$. Both curves show a typical indentation load vs depth relationship, and no indication of ferroelasticity can be seen. A gradual increase in the displacement during the holding at the maximum load was observed only for the $25 \mu \mathrm{m}$ indenter under loads above $100 \mathrm{mN}$.

Figure 8(b) shows the indentation stress vs strain curve of LSCF obtained using the sapphire indenter with radius $500 \mu \mathrm{m}$. The relationship is almost linear above the stress of $0.2 \mathrm{GPa}$, and the obtained slopes from 10 measurements were consistent above $0.2 \mathrm{GPa}$, from which the Young's modulus of LSCF was determined to be $115 \mathrm{GPa}$ (where Young's modulus and Poisson's ratio of sapphire were assumed to be 375 GPa and 0.26 , respectively). On the other hand, the slopes were significantly varied for smaller stress, especially under $0.1 \mathrm{GPa}$. Additional measurements were carefully performed to investigate the stress vs strain relationship for stress below $0.2 \mathrm{GPa}$. However, the slopes were very inconsistent and were by an order of magnitude smaller for stresses above $0.2 \mathrm{GPa}$. For example, the indentation strain varied between $0.5 \%$ and $1.2 \%$ at a stress of $0.2 \mathrm{GPa}$. The inset of Fig. $8(\mathrm{~b})$ is the result obtained using the diamond indenter with radius $25 \mu \mathrm{m}$. The indentation stress linearly increases with strain up to approximately $4.0 \mathrm{GPa}$, followed by an inflection point and another linear increase with a smaller slope. As found for the larger indenter, the indentation strain was inconsistent at the smaller stresses ( $<0.5 \mathrm{GPa}$ in this case), although the slopes for the higher stresses were consistent.

\subsection{Discussion}

A large inconsistency was observed in the indentation stress vs strain curve for stresses below $0.2 \mathrm{GPa}$ for the $500 \mu \mathrm{m}$ indenter, whereas the slope obtained for stresses above $0.2 \mathrm{GPa}$ was reproducible. Also, the obtained indentation strain was much larger than the simulated one at the lower stresses. This can be mainly 
attributed to surface roughness of the sample and varying initial contact, even though $3 \mathrm{D}$ imaging of surface roughness was performed to choose surfaces with a small roughness. Furthermore, there was no indication of ferroelasticity in the indentation stress-strain curves, which was probably be obscured by the problems associated with initial contact and surface roughness. It appears to require a material with a ferroelastic strain larger than a few $\%$ to observe the ferroelastic behaviour in the stress vs strain curve, where these problems would be neglectable in the practical application.

Young's modulus of LSCF was determined to be $115 \mathrm{GPa}$ with the $500 \mu \mathrm{m}$ sapphire indenter (determined from the stress above $0.2 \mathrm{GPa}$ ), but $189 \mathrm{GPa}$ with the $25 \mu \mathrm{m}$ diamond indenter (stress above 5 GPa). The smaller spherical indenter has a smaller contact surface area and causes higher stresses, resulting in a modulus, which is close to the unloading modulus in the uniaxial compression tests and also by sharp indentations. ${ }^{14,15}$ The small modulus obtained with the larger indenter might be affected by the problems of initial contact and surface roughness as discussed above. It should also be noted that these moduli must be smaller than the actual modulus due to the inaccurate estimation method as demonstrated in the simulation. The yield stress $\sigma_{\mathrm{Y}}$ of LSCF is evaluated to be $3.3 \mathrm{GPa}$ from the result with the $25 \mu \mathrm{m}$ indenter assuming $\sigma_{\mathrm{Yi}}$ $=1.2 \sigma_{\mathrm{Y}}$ as indicated by the elastic-plastic simulation, which is comparable to the value ( $\left.4.6 \mathrm{GPa}\right)$ determined in the previous study. ${ }^{13}$ In addition, the hardness is estimated to be $9.2 \mathrm{GPa}$ based on the relation of $H=2.8 \sigma_{\mathrm{Y}}$, which is comparable to the reported values using sharp indenters $\left(8.6 \mathrm{GPa}^{14}\right.$ and $\left.6.3 \mathrm{GPa}^{15}\right)$.

Therefore, although the simulation indicated the possibility that the initial modulus and the lower critical stress of ferroelastic materials could be experimentally obtained by examining the indentation stress vs strain curve, an improvement in accuracy of the indentation strain experiment is still required to improve the reliability of data obtained at very low displacements using large spherical indenters.

\section{Conclusion}

The present study investigated the possibility of evaluating ferroelastic mechanical characteristics by spherical indentation. Finite element simulation of spherical indentation of a ferroelastic-plastic material was performed, and the indentation load vs depth curve was obtained using characteristic bulk data of a typical 
ferroelastic oxide (LSCF). The simulated indentation stress vs strain relationship was first derived from the contact radius obtained directly from the loading simulation. Since this is not accessible in experiments, the contact radius was also estimated from the simulation of loading followed by partial-unloading as proposed by Field and Swain.

The simulation results showed that the ferroelastic-plastic mechanical behaviour cannot be observed in the indentation load vs depth curve, but is clearly observable in the indentation stress vs indentation strain curve. The simulation showed that indentation stress vs strain curve can be obtained reliably in experiments by estimating the contact radius using the loading-unloading sequence. However, the method becomes unreliable when the indentation stress exceeds the upper ferroelastic critical stress. The experiments showed that in the practical application of this technique, there is a problem in measuring sufficiently small displacements reproducibly using large radius indenting spheres in order to access the low strain region in which ferroelastic behaviour can be seen. This is partly due to surface roughness of the sample and indenting sphere and the way in which the indentation instrument detects the initial contact.

It can be concluded therefore that in principle ferroelastic mechanical characteristics could be evaluated by spherical indentation by obtaining the indentation stress vs indentation strain curve using partial unloading to estimate the contact radius. However, the requirements are very difficult to satisfy in actual experiments.

\section{Acknowledgement}

This work is supported by JSPS KAKENHI Grant Number 15KK0229. 


\section{Reference}

1. Araki W, Malzbender J. Ferroelastic deformation of $\mathrm{La}_{0.58} \mathrm{Sr}_{0.4} \mathrm{Co}_{0.2} \mathrm{Fe}_{0.8} \mathrm{O}_{3-\delta}$ under uniaxial compressive loading. J Eur Ceram Soc 2013;33:805.

2. Araki W, Abe T, Arai Y. Anomalous variation of the mechanical behaviour of ferroelastic $\mathrm{La}_{0.6} \mathrm{Sr}_{0.4} \mathrm{Co}_{0.2} \mathrm{Fe}_{0.8} \mathrm{O}_{3-\delta}$ during the ferro-to-paramagnetic transition. Scripta Mater 2013;99:9.

3. Araki W, Hanashiro D, Arai Y, and Malzbender J. Fracture mechanism of scandia-doped zirconia. Acta Mater 2013;61:3082.

4. Vullum PE, Holmestad R, Lein HL, Mastin J, Einarsrud MA, and Grande T. Monoclinic ferroelastic domains in LaCoO3-based perovskites Adv Mater 2007;19:4399.

5. Kimura Y, Tolchard J, Einarsrud MA, Grande T, Amezawa K, Hashimoto S, and Kawada T. The effect of ferroelasticity of $\mathrm{La}_{1-\mathrm{X}} \mathrm{Sr}_{\mathrm{x}} \mathrm{Co}_{1-\mathrm{Y}} \mathrm{Fe}_{\mathrm{y}} \mathrm{O}_{3-\delta}$ on the mechanical stability of solid oxide fuel cells. ECS Trans 2013;57:635.

6. Salje EKH. Ferroelastic Materials. Annu Rev Mater Res 2012;42:265.

7. Araki W, Takeda K, and Arai Y, Mechanical behaviour of ferroelastic lanthanum metal oxides $\mathrm{LaMO}_{3}$ (M=Co, Al, Ga, Fe). J Eur Ceram Soc 2016;36:4089.

8. J. Wang, S.-Q. Shi, L.-Q. Chen, Y. Li, T.-Y. Zhang, Phase field simulations of ferroelectric/ferroelastic polarization switching, Acta Materialia 2004;52:749.

9. S.C. Hwang, C.S. Lynch, R.M. Mcmeeking, "erroelectric/ferroelastic interactions and a polarization switching model, Acta metallurgica et Materialia 1995;43:2073.

10. Miura S and Kato H. Thermodynamical analysis of pseudoelasticity and calorimetry in shape memory alloys. Mater Sci Res Int 1995;1:67.

11. Miura S, Ito M, and Nakanishi N, Pseudoelastic behavior and its strain rate dependence in thermoelastic In-Tl martensite. Scripta Met 1976;10:87.

12. Orgéas L, Liu Y, and Favier D. Experimental Study of Mechanical Hysteresis of NiTi During Ferroelastic and Superelastic Deformation, J Phys IV France 1997;7:477.

13. Chen Z, Wang X, Atkinson A, and Brandon N, Spherical indentation of porous ceramics: Elasticity and 
hardness. J Eur Ceram Soc 2016;36:1435.

14. Li N, Verma A, Singh P, and Kim JH, Characterization of $\mathrm{La}_{0.58} \mathrm{Sr}_{0.4} \mathrm{Co}_{0.2} \mathrm{Fe}_{0.8} \mathrm{O}_{3-\delta}-\mathrm{Ce}_{0.8} \mathrm{Gd}_{0.2} \mathrm{O}_{2}$ composite cathode for intermediate temperature solid oxide fuel cells. Ceram Int 2013;39:529.

15. Huang BX and Malzbender J. The effect of an oxygen partial pressure gradient on the mechanical behavior of perovskite membrane Materials. J Eur Ceram Soc 2014;34:1777.

16. Muir Wood AJ and Clyne TW, Measurement and modelling of the nanoindentation response of shape memory alloys. Acta Mater 2006;54:5607.

17. Shaw GA, Stone DS, Johnson AD, Ellis AB, and Crone WC, Shape memory effect in nanoindentation of nickel-titanium thin films. Appl Phys Lett 2003;83:257.

18. Yan W, Sun Q, and Liu HY, Yan W, Sun Q, and Liu HY. Spherical indentation hardness of shape memory alloys. Mater Sci Eng A 2006;425:278.

19. Yan W, Sun Q, Feng XQ, and Qian L, Analysis of spherical indentation of superelastic shape memory alloys. Int J Solids Struct 2007;44:1.

20. Zhang Y, Cheng YT, and Grummon DS. Finite element modeling of indentation-induced superelastic effect using a three-dimensional constitutive model for shape memory Materials with plasticity. J Appl Phys 2007; 101:053507.

21. Field JS and Swain MV. A simple predictive model for spherical indentation. J Mater Res 1993;8:297.

22. Field JS and Swain MV. Determining the mechanical properties of small volumes of Material from submicrometer spherical indentations. J Mater Res 1995;10:101.

23. Johnson KL. Contact Mechanics. UK: Cambridge University Press;1985.

24. Araki W, Abe T, and Arai Y. Ferroelasticity and spin-state transitions of $\mathrm{LaCoO}_{3}$. J Appl Phys 2014;116:043513.

25. Francis HA, Phenomenological analysis of plastic spherical indentation. Trans ASME Eng Mater Technol 1976;98:272.

26. Araki W, Miyashita M, and Arai Y. Strontium surface segregation in $\mathrm{La}_{0.6} \mathrm{Sr}_{0.4} \mathrm{Co}_{0.2} \mathrm{Fe}_{0.8} \mathrm{O}_{3}-\delta$ subjected to mechanical stress. Solid State Ionics 2016;290:18. 


\section{Captions}

Table 1 Material properties used for simulations

Figure 1 Loading and partial unloading sequence: (a) Indentation load -depth curve, and (b) elastic, plastic, residual, and total deformations. The broken line indicates the fully unloaded condition.

Figure 2 Finite element simulation model of spherical indentation of disk sample: (a) The whole axisymmetric model with boundary condition at the bottom, and (b) an enlarged view of the contact area between the indenter and sample.

Figure 3 Material properties used in the finite element simulations. $E_{\mathrm{A}}, E_{\mathrm{B}}, \sigma_{\mathrm{L}}, \sigma_{\mathrm{U}}$, and $\sigma_{\mathrm{Y}}$ are initial modulus, loading modulus, lower critical modulus, upper critical stress, and yield stress, respectively. The broken lines show the unloading behaviour assuming no reverse domain switching.

Figure 4 Monotonic loading simulations with different material properties: (a) Indentation load vs depth curve, and (b) indentation stress vs strain curve. The inset of (a) shows the contact radius directly obtained in the simulation.

Figure 5 Distribution of strain energy density simulated with ferroelastic-plastic material properties $(\mathrm{N})$. Ferroelastic-domain switching and switched regions are shown in grey colour and are estimated based on a critical strain energy density of $42 \mathrm{~kJ} / \mathrm{m}^{-3}$.

Figure 6 Loading-partial unloading simulation of an elasto-plastic material: (a) Indentation load and depth curve, and (b) indentation stress and strain curve. The inset of (a) compares the contact radii obtained directly with those estimated from the partial unloading simulations.

Figure 7 Loading-partial unloading simulation of a ferroelastic-plastic material: (a) Indentation load vs depth curve, and (b) indentation stress vs strain curve. The inset of (a) compares the contact radii obtained directly with those estimated from the partial unloading simulations.

Figure 8 Experimental spherical indentation of dense LSCF using the sapphire indenter with radius 500 $\mu \mathrm{m}$ : (a) Indentation load vs depth curve, and (b) indentation stress vs strain curve. The insets are the results obtained using the diamond indenter with radius $25 \mu \mathrm{m}$. 
Table 1 Material properties used for simulations

\begin{tabular}{|c|c|c|c|c|c|c|c|c|c|}
\hline \multirow{2}{*}{ Materials/Properties } & & $v_{\mathrm{i}}$ & $E$ & $v$ & $\sigma_{Y}$ & $E_{\mathrm{A}}$ & $E_{\mathrm{B}}$ & $\sigma_{L}$ & $\sigma_{U}$ \\
\hline & $(\mathrm{GPa})$ & & $(\mathrm{GPa})$ & & (MPa) & $(\mathrm{GPa})$ & $(\mathrm{GPa})$ & $(\mathrm{MPa})$ & (MPa) \\
\hline Elastic & \multirow{4}{*}{1141} & \multirow{4}{*}{0.07} & \multirow{2}{*}{150} & \multirow{2}{*}{0.3} & - & - & - & - & - \\
\hline Elasto-plastic & & & & & \multirow{2}{*}{4600} & - & - & - & - \\
\hline Ferroelastic-plastic (N) & & & - & - & & 150 & 200 & 105 & 237 \\
\hline Ferroelastic-plastic (M) & & & Disc & 1. & points & from $\mathrm{Fi}$ & 3 wer & ed as in & ut data. \\
\hline
\end{tabular}



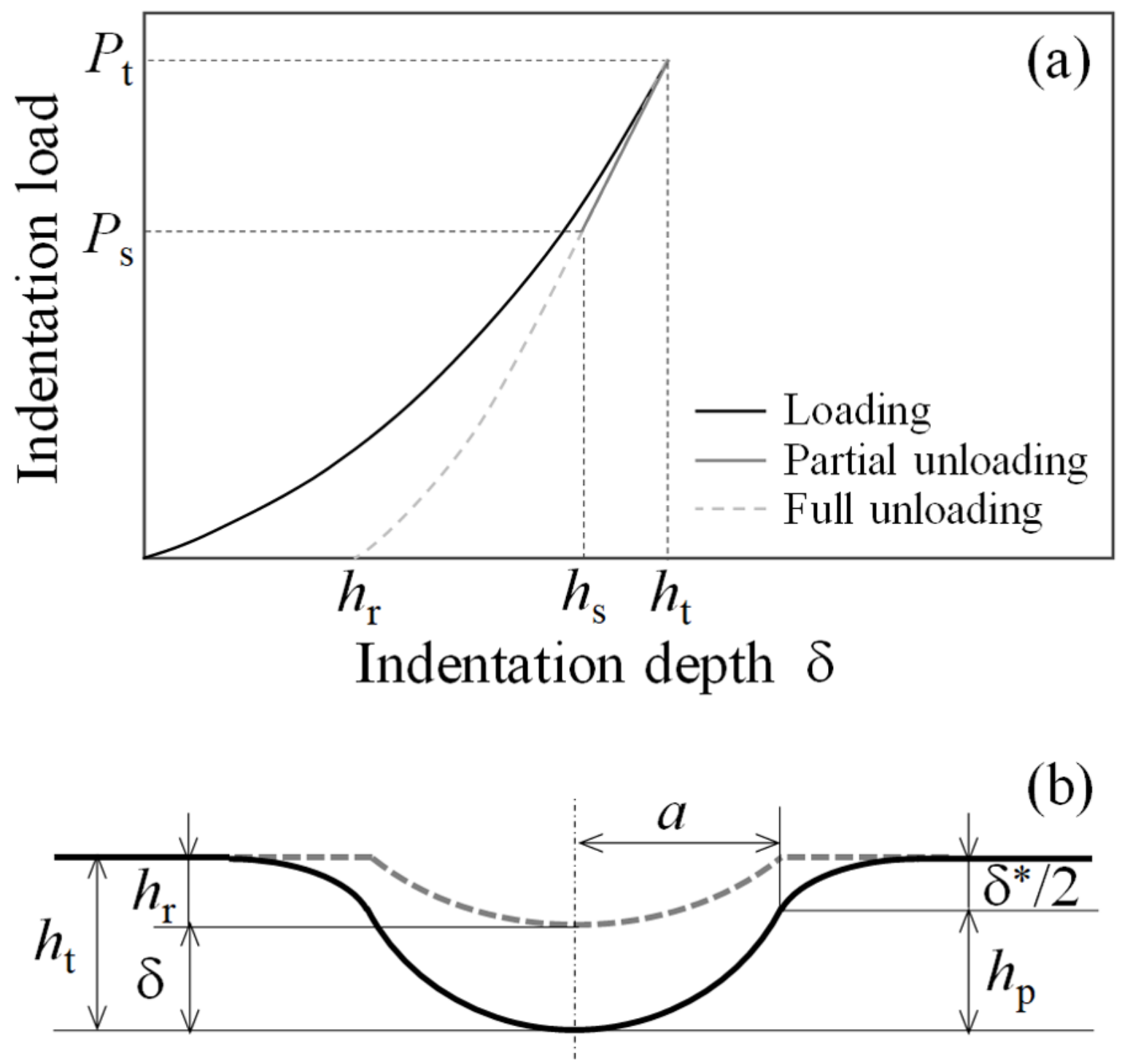

Figure 1 Loading and partial unloading sequence: (a) Indentation load -depth curve, and (b) elastic, plastic, residual, and total deformations. The broken line indicates the fully unloaded condition. 


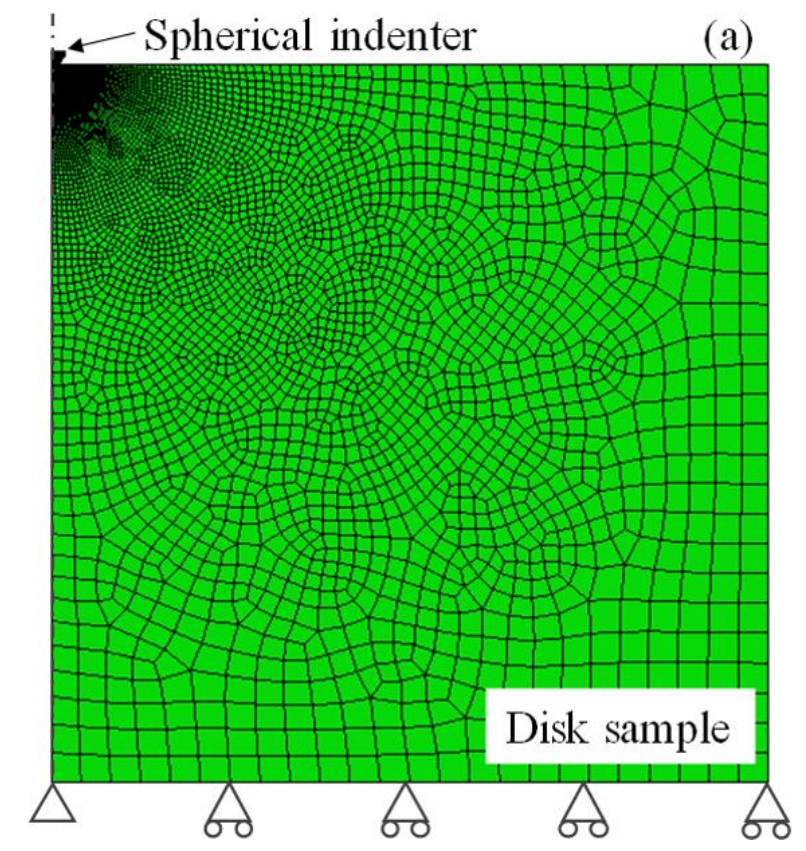

(b)

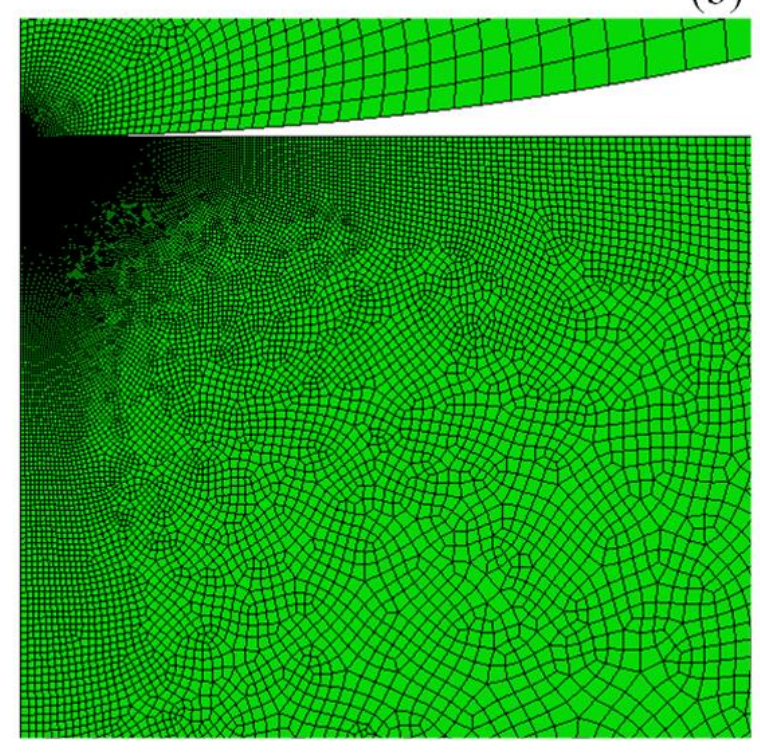

Figure 2 Finite element simulation model of spherical indentation of disk sample: (a) The whole axisymmetric model with boundary condition at the bottom, and (b) an enlarged view of the contact area between the indenter and sample. 


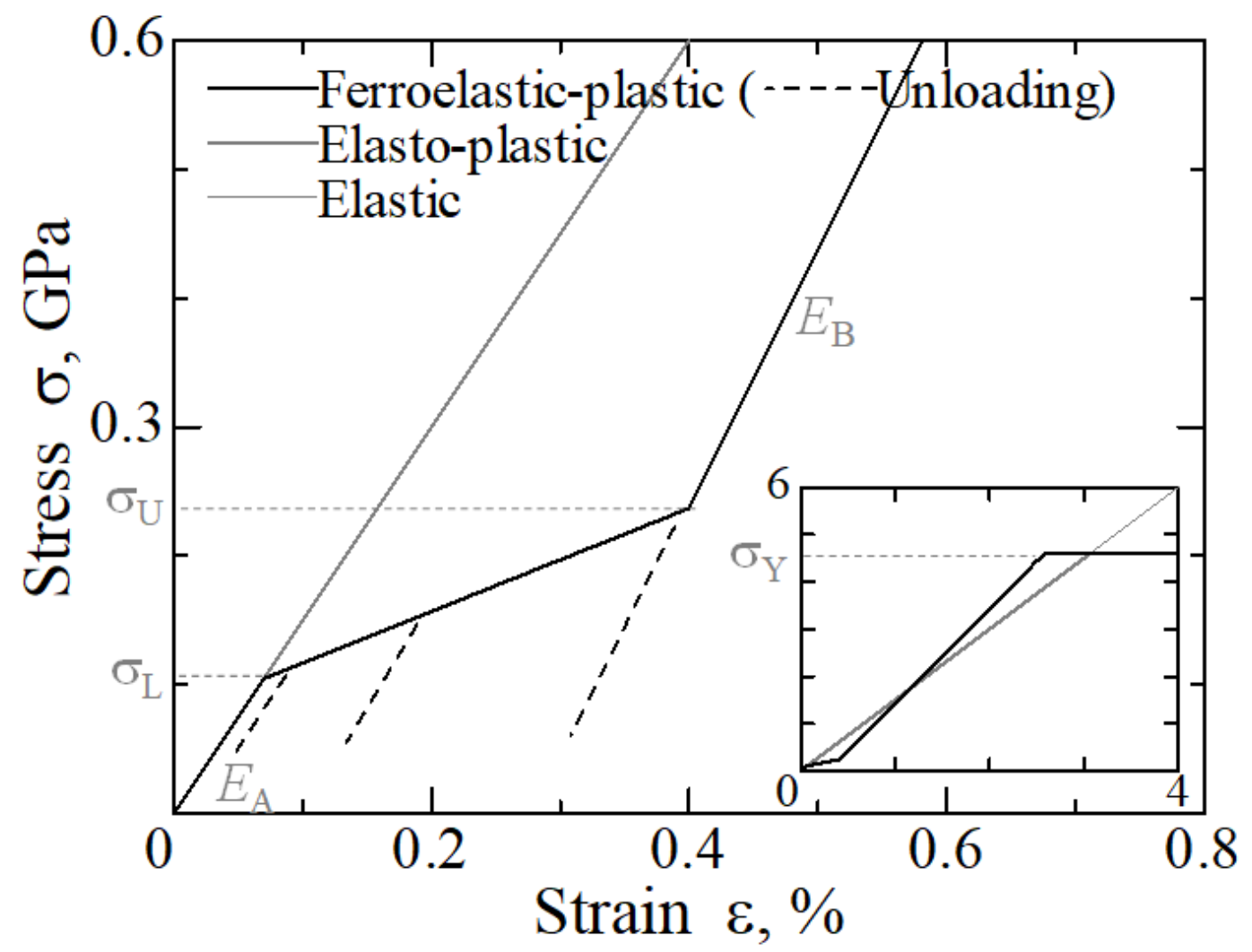

Figure 3 Material properties used in the finite element simulations. $E_{\mathrm{A}}, E_{\mathrm{B}}, \sigma_{\mathrm{L}}, \sigma_{\mathrm{U}}$, and $\sigma_{\mathrm{Y}}$ are initial modulus, loading modulus, lower critical modulus, upper critical stress, and yield stress, respectively. The broken lines show the unloading behaviour assuming no reverse domain switching. 

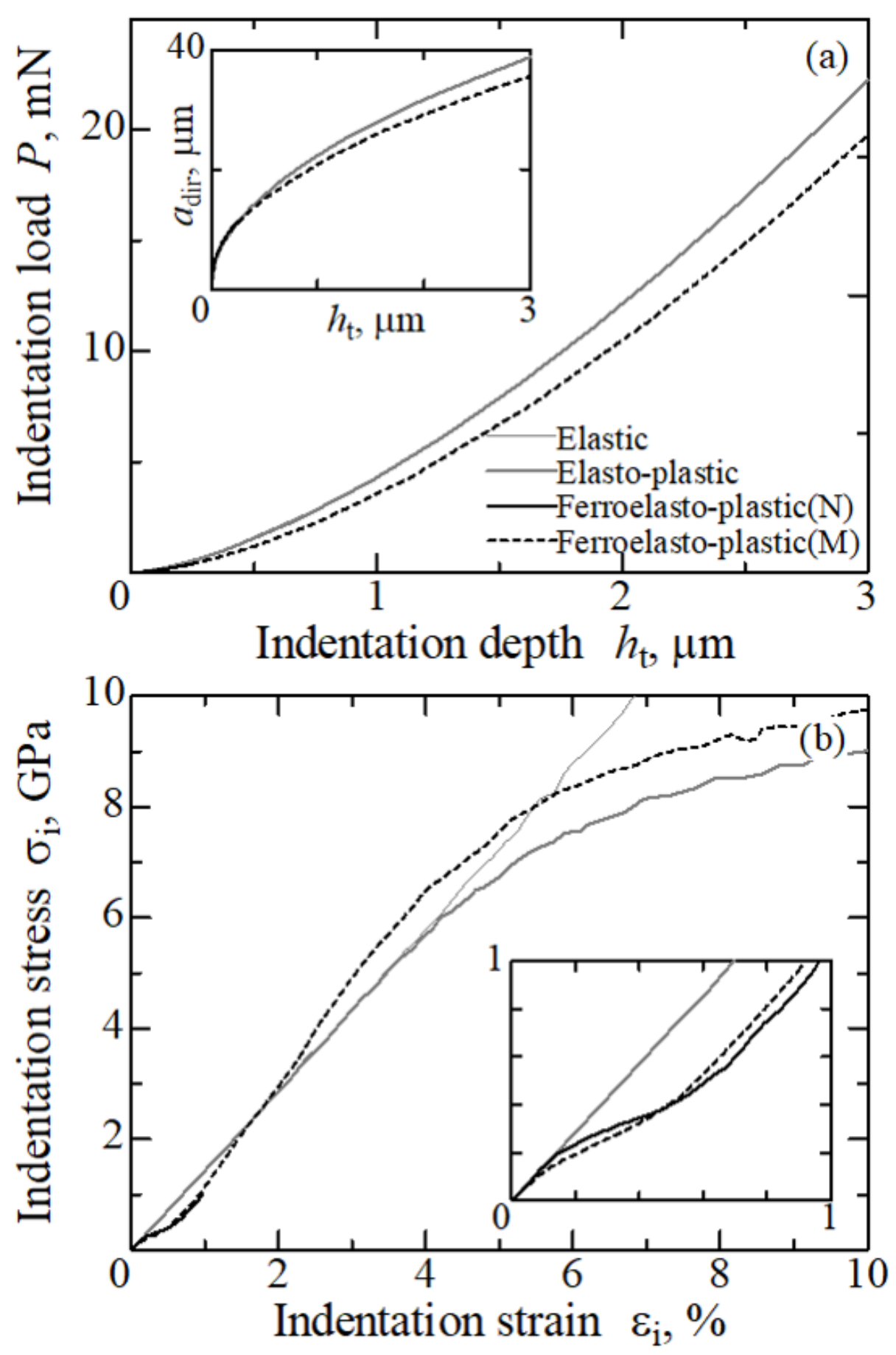

Figure 4 Monotonic loading simulations with different material properties: (a) Indentation load vs depth curve, and (b) indentation stress vs strain curve. The inset of (a) shows the contact radius directly obtained in the simulation. 

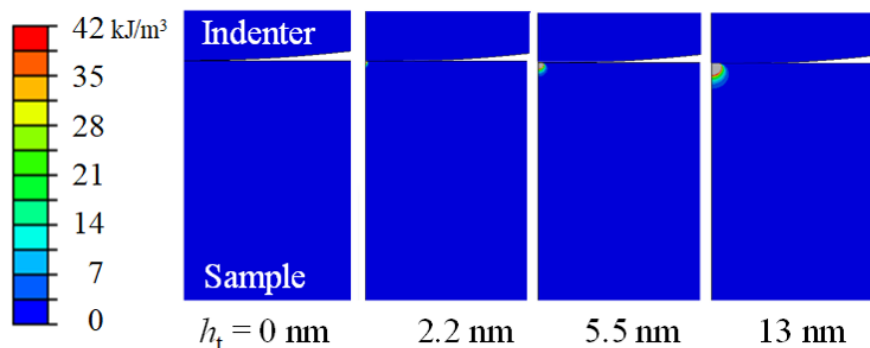

$h_{\mathrm{t}}=0 \mathrm{~nm}$

$2.2 \mathrm{~nm}$

$5.5 \mathrm{~nm}$

$13 \mathrm{~nm}$

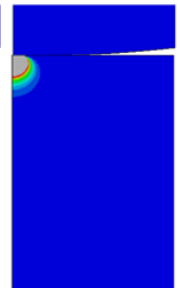

$26 \mathrm{~nm}$

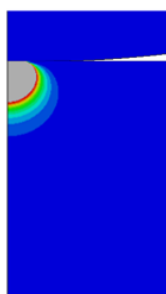

$59 \mathrm{~nm}$

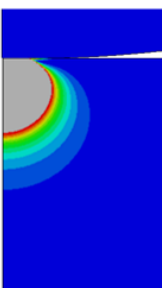

$124 \mathrm{~nm}$

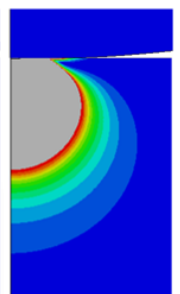

$204 \mathrm{~nm}$

Figure 5 Distribution of strain energy density simulated with ferroelastic-plastic material properties $(\mathrm{N})$.

Ferroelastic-domain switching and switched regions are shown in grey colour and are estimated based on a critical strain energy density of $42 \mathrm{~kJ} / \mathrm{m}^{-3}$. 

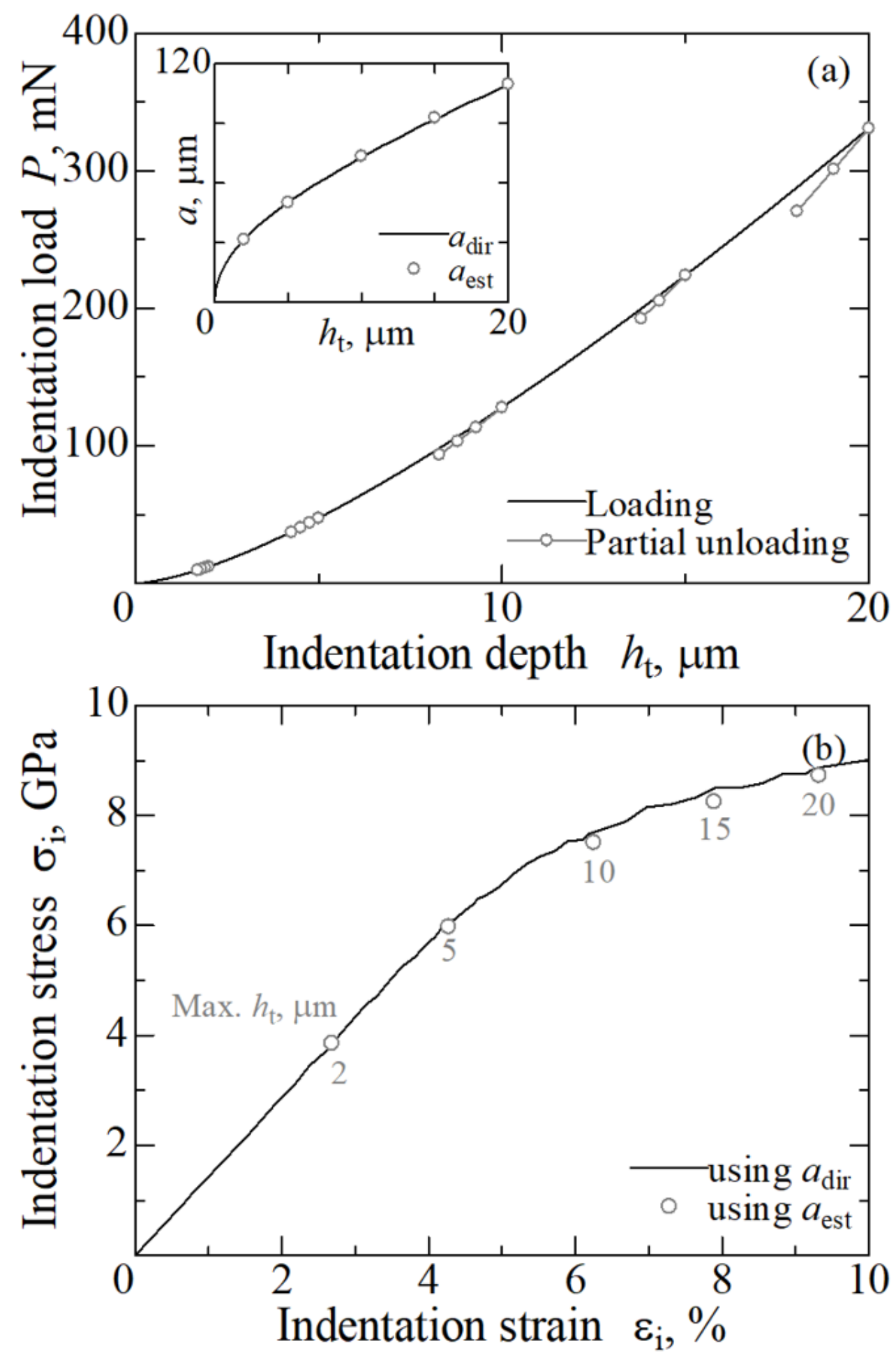

Figure 6 Loading-partial unloading simulation of an elasto-plastic material: (a) Indentation load and depth curve, and (b) indentation stress and strain curve. The inset of (a) compares the contact radii obtained directly with those estimated from the partial unloading simulations. 

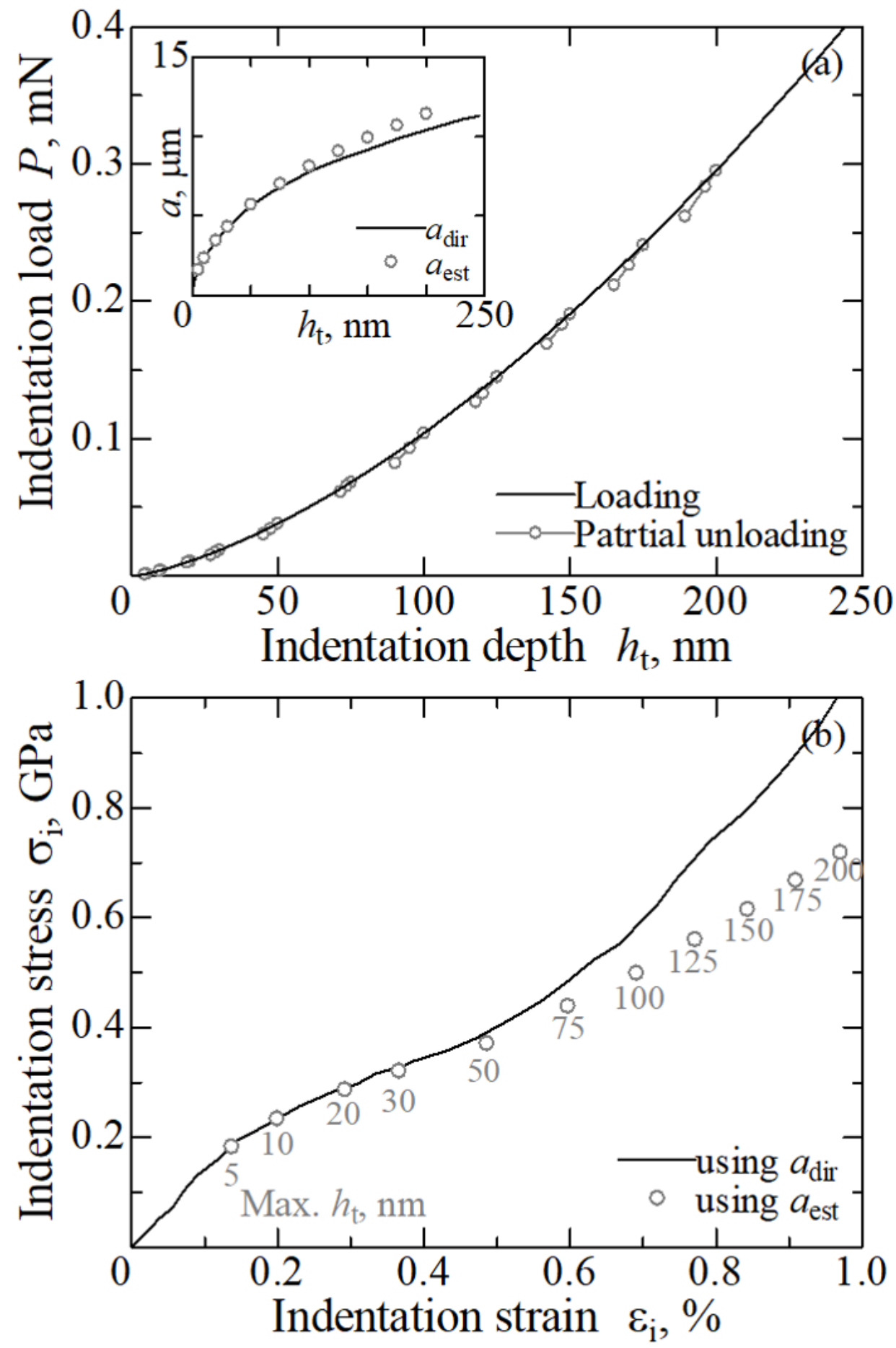

Figure 7 Loading-partial unloading simulation of a ferroelastic-plastic material: (a) Indentation load vs depth curve, and (b) indentation stress vs strain curve. The inset of (a) compares the contact radii obtained directly with those estimated from the partial unloading simulations. 

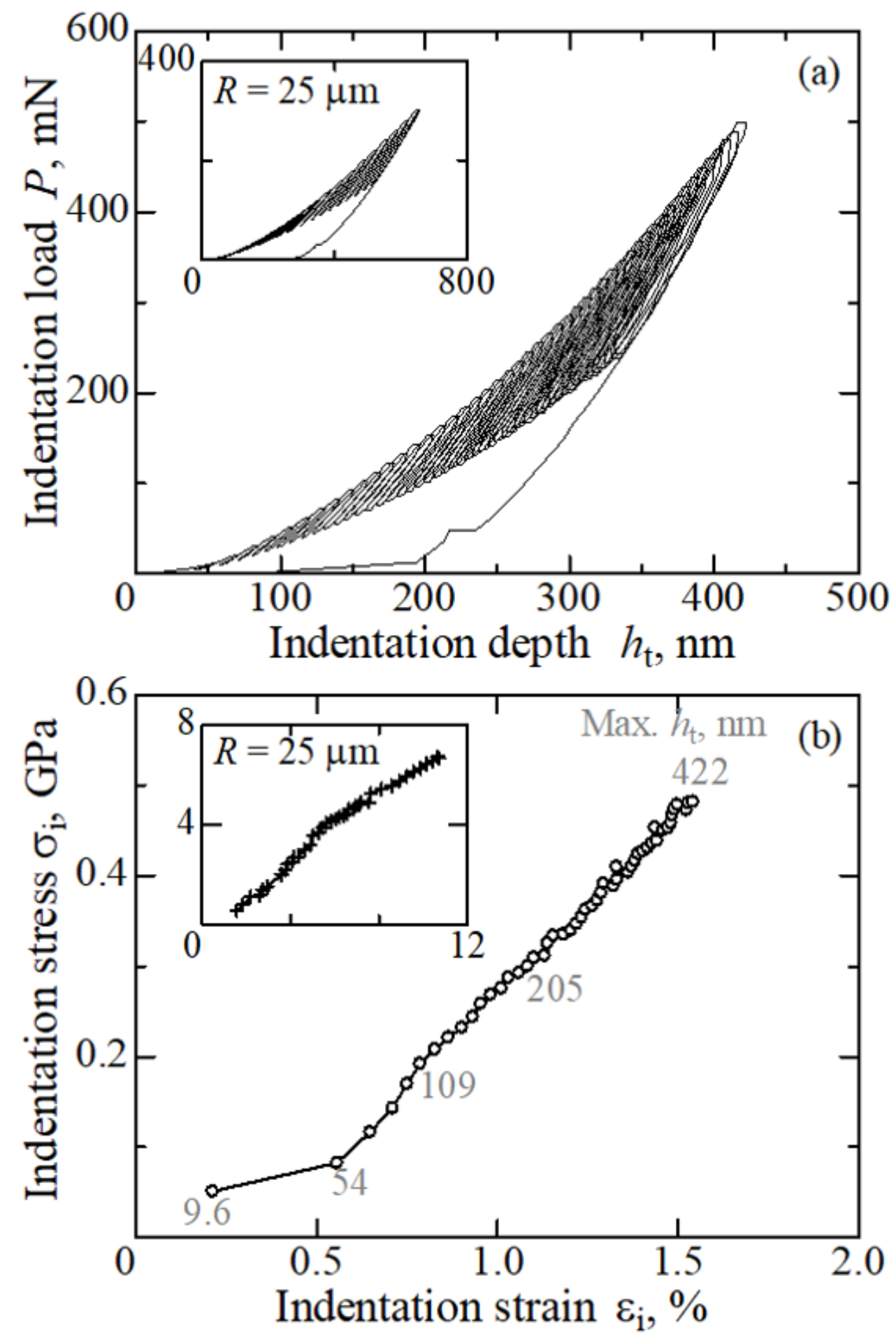

Figure 8 Experimental spherical indentation of dense LSCF using the sapphire indenter with radius 500 $\mu \mathrm{m}$ : (a) Indentation load vs depth curve, and (b) indentation stress vs strain curve. The insets are the results obtained using the diamond indenter with radius $25 \mu \mathrm{m}$. 